\title{
INFLAMMATORY STATUS AND ASYNCHRONOUS BIOMARKER STORM ARE ASSOCIATED WITH LACK HUMORAL RESPONSE AFTER 17DD-YELLOW FEVER PRIMARY VACCINATION IN AUTOIMMUNE DISEASES
}

Ketty Lysie Libardi Lira Machado일 Ismael Artur da Costa Rocha², Sávio Carvalho Deotti ${ }^{1}$, Samira Tatiyama Miyamoto ${ }^{1}$, Priscila Costa Martins Rocha $^{1}$, Erica Vieira Serrano ${ }^{1}$, Valquiria Garcia Dinis ${ }^{1}$, Sônia Alves Gouvêa ${ }^{1}$, João Gabriel Fragoso Dias ${ }^{1}$, Ana Carolina Campi-Azevedo², Andréa Teixeira-Carvalho' ${ }^{2}$, Vanessa Peruhype-Magalhães ${ }^{2}$, Maria Bernadete Renoldi de Oliveira Gavi ${ }^{1}$, Lidia Balarini da Silva ${ }^{1}$, Ruben Horst Duque ${ }^{1}$, Ana Paula Espíndula Gianordoli ${ }^{1}$, Thays Zanon Casagrande ${ }^{1}$, Karine Gadioli Oliveira ${ }^{1}$, Maria de Fatima Bissoli ${ }^{1}$, Maria da Penha Gomes Gouvea ${ }^{1}$, Sheila Maria Barbosa de Lima ${ }^{3}$ Emily Hime Miranda³ ${ }^{3}$ Gisela Freitas Trindade ${ }^{3}$, Olindo Assis Martins-Filho², Valéria Valim Cristo ${ }^{1, \star}$

1.Universidade Federal do Espírito Santo, Vitória (ES), Brazil. 2.Fundação Oswaldo Cruz, Belo Horizonte (MG), Brazil. 3.Fundação Oswaldo Cruz, Rio de Janeiro (RJ), Brazil.

*Corresponding author:val.valim@gmail.com

\section{BACKGROUND}

Recent evidence showed that planned 17DD-YF primary vaccination is safe and immunogenic, but lower seropositivity rate in patients with autoimmune diseases (AID). The objective of this study was to investigate biomarkers of immune response in AID comparing those with positive and negative neutralizing antibodies.

\section{MATERIALS AND METHODS}

This is a phase IV controlled prospective study, including AID patients and healthy controls, vaccinated for the first time with the 17DD YF vaccine. Included patients were referred for planned vaccination by a rheumatologist, in remission or with low disease activity and low-level immunosuppression. The kinetic timelines of the plaque reduction neutralization test (PRNT), seropositivity rates (PRNT $\geq 1: 50$ ), 17DD-YF viremia and serum biomarkers [IL-1beta, IL-1ra, IL-2, IL-4, IL-5, IL-6, IL-7, IL-8, IL-9, IL-10, IL-12 (p70), IL-13, IL-15, IL-17, eotaxin, basic FGF, G-CSF, GM-CSF, IFN-gamma, IP-10, MCP- 1 (MCAF), MIP-1alpha, MIP-1beta, PDGF-BB, RANTES, TNF-alpha, VEGF] after 17DD-YF primary vaccination were evaluated at various time points.

\section{RESULTS}

Individuals evaluated $(n=278)$, including patients with rheumatoid arthritis ( $R A=79)$, spondyloarthritis $(S p A=59)$, systemic sclerosis ( $\mathrm{SSC}=8$ ), systemic lupus erythematosus ( $S L E=27)$, primary Sjögren's syndrome $(S S=54)$ and healthy controls $(H C=51)$ (Fig. 1). Only mild adverse events were reported. Seropositivity rate (78 vs. 96\%, $p=0.01$ ) were lower in patients with AID after 28 days compare to HC (Fig. 2). Viremia kinetic was similar in both groups. In general, the 17DD-YF vaccine triggered a more prominent serum biomarker response in AID as compared to HC. Moreover, AID patients with PRNT positive (AID/PRNT+) presented an earlier serum biomarker response with higher fold values at D3-4/D5-6 as compared to AID with PRNT negative (AID/PRNT-) that presented higher fold values at late time-points (D7 and D14-D28) (Fig. 3). Autoimmune diseases with PRNT negative presented higher levels of some biomarkers at baseline (CXCL8, CCL11, CXCL10, TNF a, IL15, IL17, IL10, G-CSF, GMCSF) suggesting that inflammatory status prior vaccination may contribute to the lack of antibody response. The IFN-gamma level is elevated in HC, AID and AID/PRNT+ groups, but low in AID/PRNT- at the day of peak viremia (D5-6). Interestingly, in AID/PRNT+ group, biomarkers tend to deflate in postvaccination highlighting an immunomodulatory effect of vaccine. (Fig. 4). 


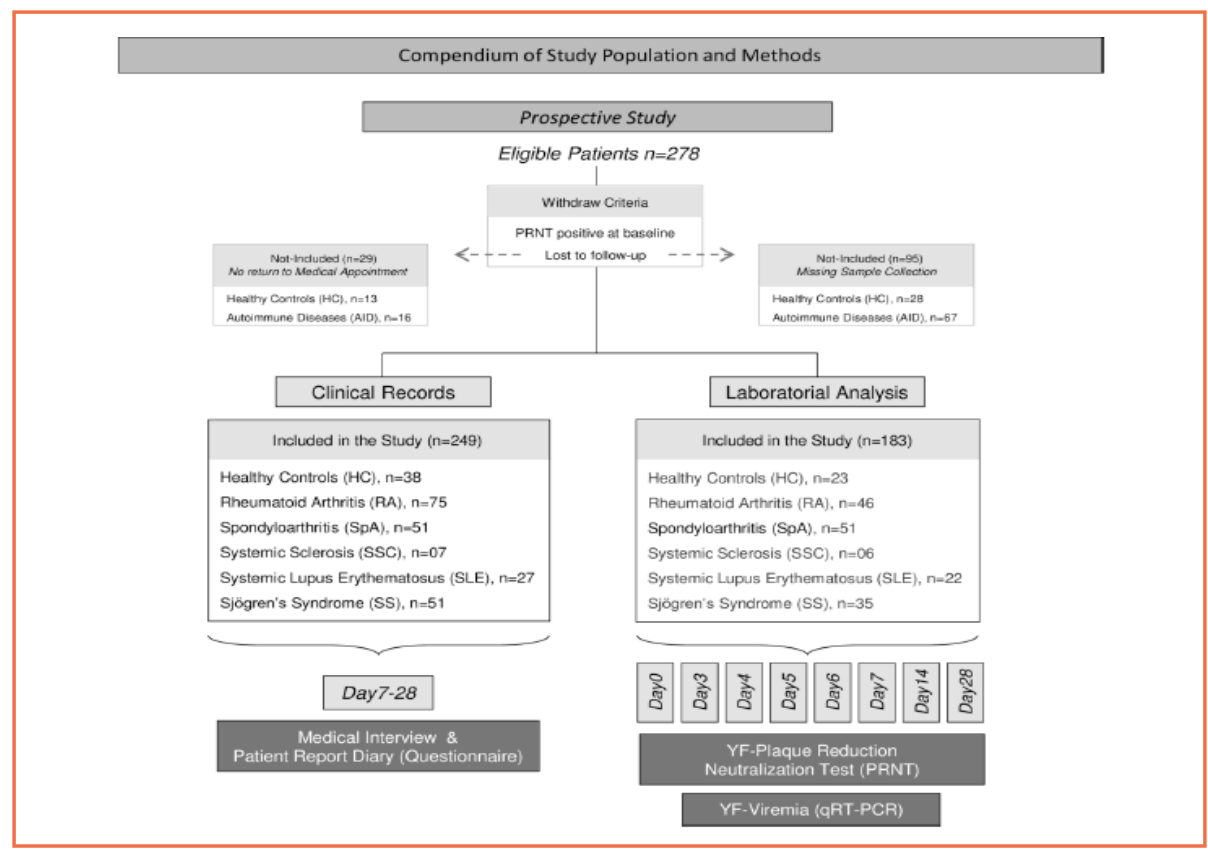

Figure 1. Overview of the study population and methods. Laboratory analyses includes YF-plaque reduction neutralization test (PRNT) and YF viremia (RNAnemia) analysis by qRT-PCR.

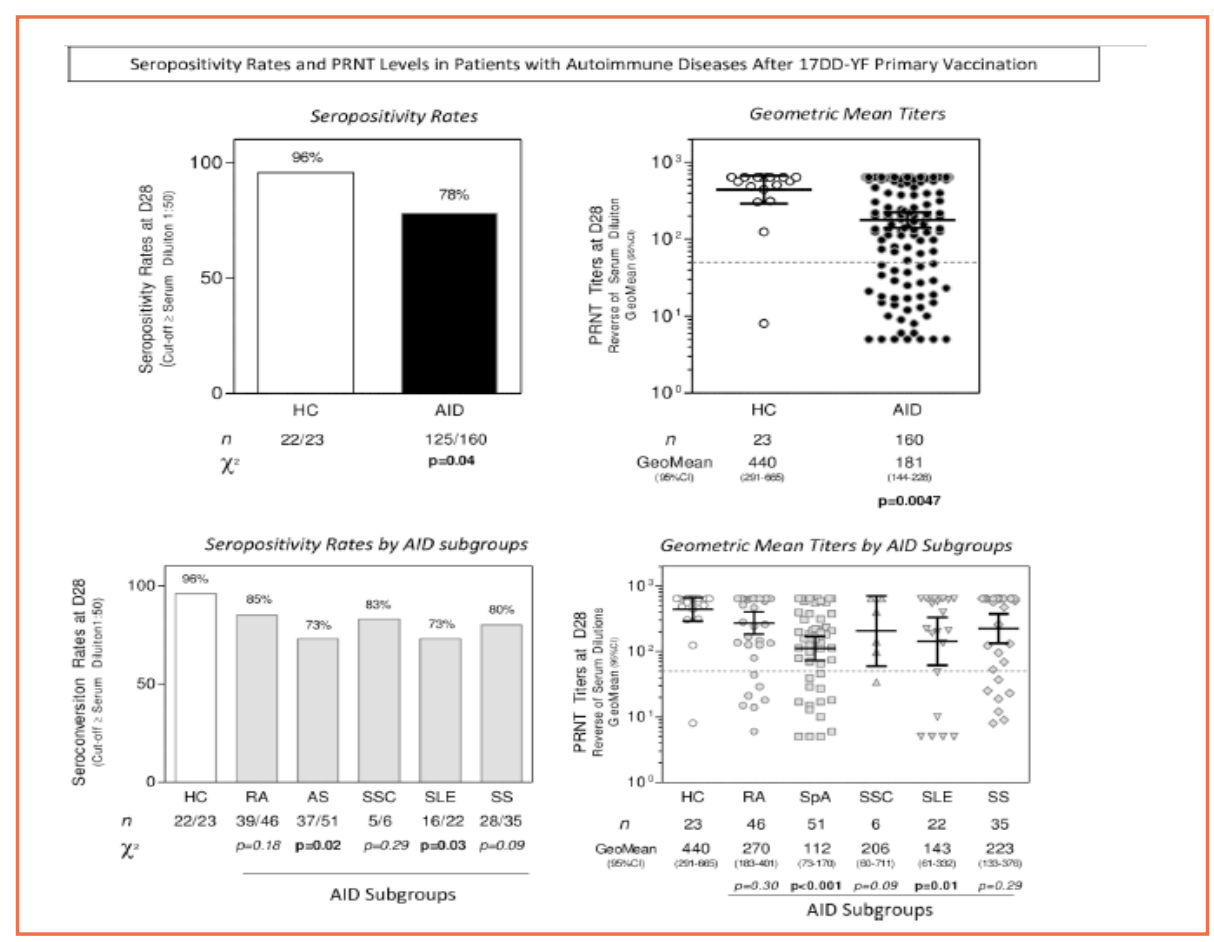

Figure 2. Seropositivity rates and PRNT levels after 17DD-YF primary vaccination in patients with AID. 


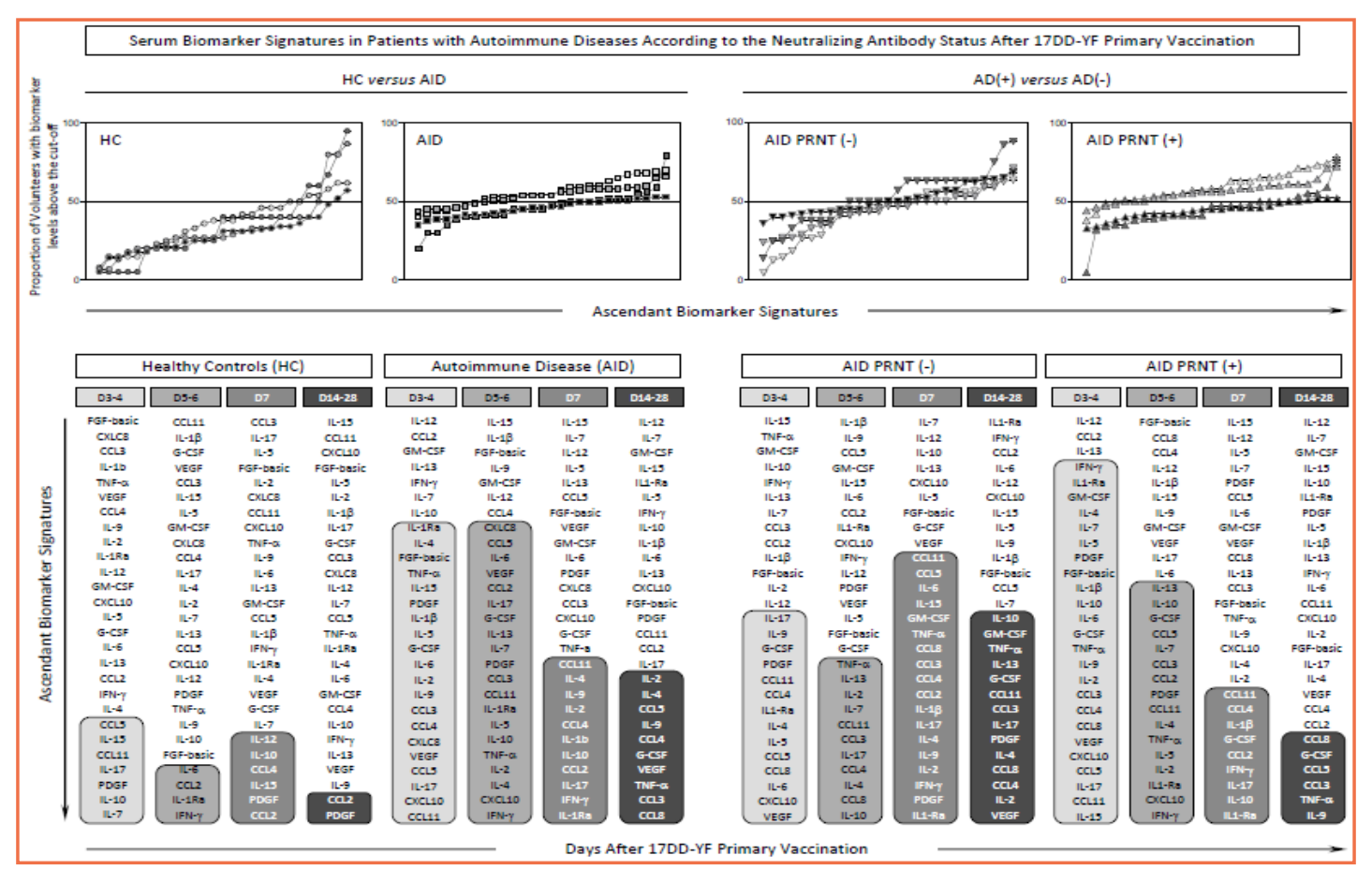

Figure 3. Serum biomarker signatures in patients with autoimmune diseases according to the neutralizing antibody status after 17DD-YF primary vaccination.

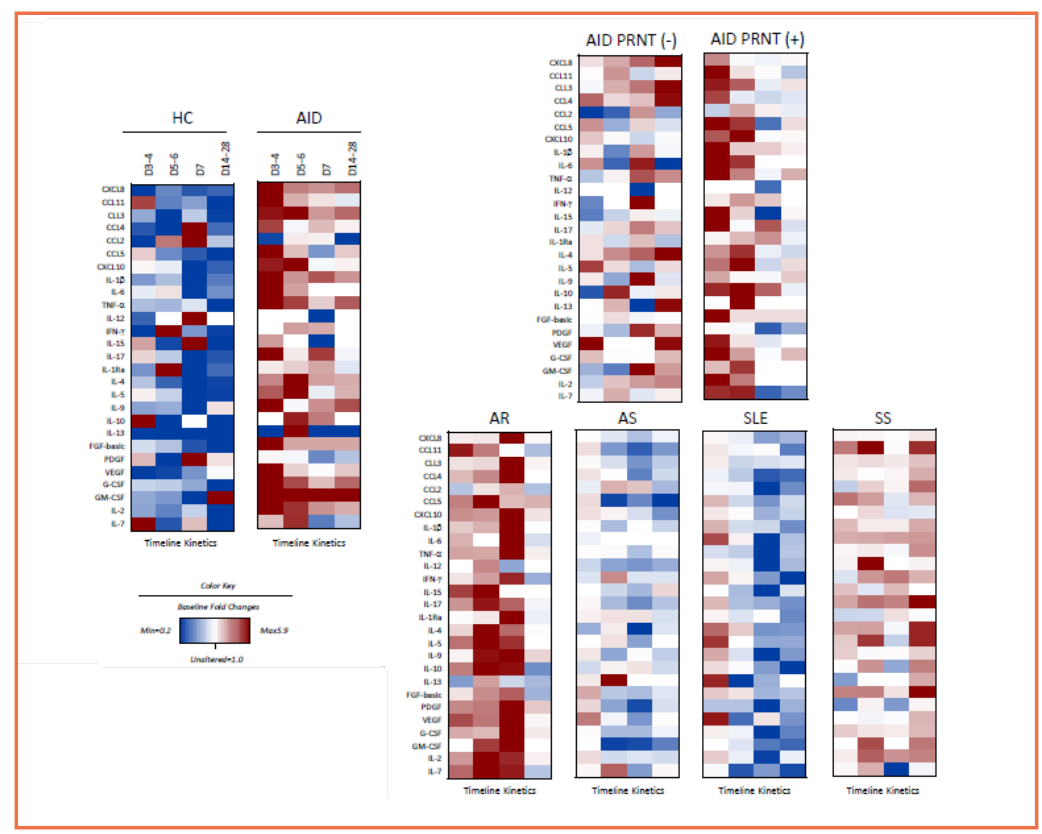

Figure 4. Kinetics of serum biomarker signatures after primary 17DD-YF vaccination according to the neutralizing antibody status type of autoimmune disease.

\section{CONCLUSION}

These data suggested that inflammatory status prior vaccination, low INF-gamma at viremia peak and the occurrence of asynchronous biomarker storm after 17DD-YF vaccination are somehow associated with lack of antibody response. 